\title{
Auditory Biofeedback Decreases Jump Performance In Figure Skaters
}

\author{
JOAO A. C. BARROS ${ }^{1}$ | LLANEL FLORENDO | YVONNE LE ${ }^{1,2}$ | \\ 1Department of Kinesiology, California State University Fullerton, Fullerton, California, USA. \\ ${ }^{2}$ Stanbridge College, Irvine, California, USA
}

Correspondence to: Joao A. C. Barros, 230 Kinesiology and Health Sciences Bldg., 800 N. State College Blvd., Fullerton, CA 92831, USA. email: jbarros@fullerton.edu

\begin{abstract}
AT A GLANCE
The few studies that have attempted to produce increase in jump height in figure skaters have failed to do so. The current study attempted to achieve that goal using auditory biofeedback. Though increased jump height was not identified, the results confirmed previous studies in that auditory biofeedback led to changes in the observed movement pattern.
\end{abstract}

INTRODUCTION: The few studies that attempted to produce increase in jump height in figure skaters ${ }^{4,5}$ have failed to do so. These studies did not focus on increasing knee flexion, a critical factor for jump height. ${ }^{15,16}$ Auditory biofeedback has been shown to modify posture, balance and cycling performance ${ }^{11,13,14}$ and could potentially be used to increase knee flexion in figure skaters.

PURPOSE: To investigate the effects of auditory biofeedback on the performance of Lutz jumps.

METHODS: Thirteen intermediate level female adolescence figure skaters performed 6 off-ice Lutz jumps under each of 2 conditions: 1) WITH auditory biofeedback; 2) and WITHOUT auditory biofeedback. Auditory biofeedback was provided via EMG Retrainer. Separate repeated measures ANOVAs were conducted for time in the air, knee flexion and EMG activity.

RESULTS: Differences between conditions for time in the air $(p=.012)$ and knee flexion $(p=.049)$ were identified.

CONCLUSION: Auditory biofeedback increased knee flexion and decreased jump height. In this case, auditory biofeedback might have directed performers' attention to an internal cue disrupting performance.

\section{INTRODUCTION}

The competitive nature of figure skating has led the sport towards increased athleticism. In this context, figure skaters must perform a higher number of revolutions during a jump. To achieve that goal, the athlete must increase vertical velocity and angular momentum at take-off and decrease moment of inertia while in flight. ${ }^{1}$ King et al. (2004) described the characteristics of the quadruple toe-loop jumps in comparison to the triple toe-loop jumps performed by 19 male figure skaters in the 2002 Winter Olympics. Their analysis of the propulsion phase and take-off indicated that subjects who completed triple toe-loop jumps as a "warm-up" to perform the quadruple toe-loop jump and subjects who completed quadruple toe-loop jumps had higher vertical velocity compared to skaters who performed the triple toe-loop jumps with no attempt of performing the quadruple toe-loop jump. The authors additionally suggested that the ability to achieve appropriate vertical velocity and consequently complete the revolutions in a jump depends greatly on the technique at takeoff.

Albert \& Miller (1996) provide further evidence that supports the link between vertical velocity and takeoff technique. After investigating the characteristics of the glide, transition, and pivot portions of takeoff in single and double axel jumps, the authors suggested that skid performed during the transition phase may be the factor limiting angular momentum needed to complete the revolutions off the ice. These results led the authors to argue that without the skid, the increase in vertical velocity could potentially produce the jump height needed to complete the desired revolutions during flight. 
Though takeoff technique is central to increased jump height, the few studies that have attempted to improve figure skaters' jumping techniques have failed to demonstrate positive results. For example, Law \& Ste-Marie (2005) investigated whether self-modeling would have an additional effect to physical practice in skaters' jump performance and selfefficacy, motivation, and state anxiety. Nineteen intermediate female figure skaters were assigned to videotape self-modeling with physical practice group, physical practice only group, or control group. No significant differences in performance or psychological variables were identified. Haguenauer et al. (2005) studied whether different types of instruction (i.e. sub goal of the task or metaphor) enhanced learning of the bunny-hop. Eighteen novice skaters, with one to two years of skating experience and who currently skated two hours per week, were chosen to participate in the study. Participants were divided into three groups: demonstration and sub goal instructions, demonstration and metaphor instructions, and demonstration only. Performance was evaluated in terms of jump height, jump length and thigh and hip angles. The different instructions did not lead to improved performance in terms of jump height or length, but participants in the demonstration and metaphor instructions group had higher hip angle at takeoff.

A possible technique to improve learners jump height is the use of auditory biofeedback. Auditory biofeedback is a tool that allows the learner insight to his or her own physiological processes. Auditory biofeedback about the learner's muscle activity could be displayed and heard, thereby increasing proprioception. ${ }^{6}$ The use of auditory biofeedback has been shown to affect postural sway and balance $7,8,9,10,11,12,13$ and cycling performance in children. ${ }^{14}$ Therefore, the purpose of this study is to investigate the effects of audio biofeedback on the jump height of figure skaters. It is hypothesized that audio biofeedback will increase the jump height and knee flexion during the off-ice Lutz jump.

\section{METHODS}

\section{Participants}

Twenty adolescent female figure skaters (14-17 years of age) volunteered for the study. US Figure Skating uses a series of structured tests in order to determine a skater's skill level category. These categories are used to promote balanced competition. In addition to skill level, these categories include age restrictions. In this study, all skaters were in the intermediate category. Skaters in this category can complete a single Lutz jump (i.e. complete rotation and land on one foot without losing balance) with strong smooth edges and turns combined with correct posture and effortless flow. All skaters volunteered to be in the study with assent and written informed consent from parent or legal guardian. All participants had two or more years of experience in competitive figure skating, ability to complete a single Lutz jump, and were currently developing their ability to perform axel and/or double jumps. The participants practiced a minimum of two times a week for an hour at an ice skating facility in Southern California. All skaters in this facility who were in the intermediate level and met the study inclusion criteria were part of study. The university's Institutional Review Board approved the following procedures.

\section{Task}

Participants were asked to perform the Lutz jump off of the ice. The actual Lutz jump is a foundational maneuver that is mastered before the development of more advanced jumps. 
The skater moves backwards on the outside edge of the support foot, leans forward while extending the opposite leg backwards and bending the knee of the support leg in preparation for takeoff, then moves the opposite leg towards the ice to plant the toe into the ice. As the takeoff leg picks the ice, it initiates the jump and causes rotation. After take off, the skater makes one full rotation before landing on the outside edge of the same skate that picks into the ice, still moving in a backward direction. In the off-ice Lutz jump participants start and end the jump in a static position standing on the support leg. Participants wore unrestrictive clothing and sneakers. The rest of the movement was performed as the actual Lutz jump. A successful trial consisted of the completion of the rotation and the ability to land on one leg without losing balance.

\section{Procedure}

Data collection was completed in one session lasting approximately one hour. Participants were instructed to wear athletic pants regularly worn during practice sessions. After parents or legal guardians provided written informed consent, and after assent from each participant was obtained, the goal of the experiment, the experimental procedures, and the equipment were explained to the participants.

A video camera and the EMG Retrainer (Chattanooga Group Inc.) were used to record the performance and provide auditory biofeedback, respectively. The video camera was placed on a fixed tripod at 90 degrees laterally, 1.52 meters from the takeoff location to obtain a lateral view of the Lutz performance. The EMG Retrainer sensor (i.e. electromyography sensor) was placed on the rectus femoris of the participants' support leg. The EMG Retrainer produced a loud beep when participants achieved target muscle activation, which was determined for each participant by observing each participant's muscle activation during a static position of 70 degrees of knee flexion while in the single Lutz position for takeoff. A loud beep was produced when the muscle activation of the rectus femoris was within a 20 percent margin of that value.

Following the placement of the EMG Retrainer and the determination of muscle activation target, participants were given a 2-minute warm-up to become familiarized with the equipment and camera set up. Participants were then asked to perform six trials under each of the two conditions: WITH auditory biofeedback and WITHOUT auditory biofeedback. In the trials WITH auditory biofeedback participants were told to "complete the Lutz jump as well as possible; you will hear a beep when you reach the correct knee angle for the jump". In the trials WITHOUT auditory biofeedback participants were told to "complete the Lutz jump as well as possible; and remember to flex the knee at 70 degrees before you jump." The order in which participants performed the conditions was counterbalanced. The second set of trials was completed after a five-minute rest period during which participants watched a movie clip as a "wash out" period for removing the effects of the first condition's effects. Each trial consisted of performing the off-ice single Lutz jump. Participants performed 12 trials, six under each condition. If a trial was not successful, (i.e. participants lost balance or did not complete the rotation) it would be repeated. However, none of the participants had to repeat a trial.

\section{Treatment of the Data}

Time in the air, knee flexion and peak muscle activity (i.e. maximum EMG activity observed in millivolts) were analyzed. Time in the air and knee flexion were obtained through video analysis using Dartfish software. For Lutz jumps on ice, time in the air 
begins when the pick of the skate leaves the ice. Since in this study the jump was performed off-ice, the apex of the toe part of the shoe was operationally defined as the pick of the skate. Peak muscle activity was obtained through the EMG Retrainer. Scores for each of the dependent variables (i.e. time in the air, knee flexion and peak muscle activity) were averaged for each participant across each of the audio biofeedback conditions: WITH audio biofeedback and WITHOUT audio biofeedback.

Statistical analysis of the average scores was performed using SPSS v.10. Separate one-way repeated measures analyses of variance were conducted for each of the dependent variables (i.e. time in the air, knee flexion, and peak muscle activity of the rectus femoris). When appropriate, $F$ ratios involving repeated-measures factors were reported with the Greenhouse-Geisser $\mathrm{df}$ adjustment. Partial eta-squared values $\left(\eta_{\mathrm{p}}{ }^{2}\right)$ were also reported to indicate effect sizes for significant results. For all analyses, a was set at .05 .

\section{RESULTS}

\section{Time in the air}

Mean time in the air during trials WITH auditory biofeedback was $0.332+0.042 \mathrm{~s}$, while mean time in the air during trials WITHOUT auditory biofeedback was $0.348+0.046 \mathrm{~s}$. The result of the repeated measures ANOVA determined that mean time in the air was statistically significant different between conditions $\left(F(1,9)=9.912, p=.012, \eta_{p}^{2}=.524\right)$.

\section{Knee flexion}

Knee flexion during trials WITH auditory biofeedback was $116.08+6.08$ degrees, while knee flexion during trials WITHOUT auditory biofeedback was $113.1 \overline{8}+6.51$ degrees. The statistical analysis confirmed the significant difference between conditions $(F(1,10)=$ 4.997, $p=.049, \eta_{p}^{2}=.333$ ).

\section{EMG activity}

Mean EMG activity during trials WITH auditory biofeedback was $565.38+212.13 \mathrm{mV}$, while mean EMG activity during trials WITHOUT auditory biofeedback was $590.40+179.97 \mathrm{mV}$. The statistical analysis indicated that mean EMG activity did not differ significantly between conditions $\left(F(1,11)=.945, p=.342, \eta_{p}^{2}=.079\right)$.

Table 1: Means $( \pm$ SD) of dependent variables in trials WITH auditory biofeedback and WITHOUT auditory biofeedback.

\begin{tabular}{cccc}
\hline Condition & Time in the air (s) & Knee Flexion (degrees) & EMG Activity (mV) \\
\hline WITH & $0.332+0.042$ & $116.08+6.08$ & $565.38+212.13$ \\
\hline WITHOUT & $0.348+0.046$ & $113.18+6.51$ & $590.40+179.97$ \\
\hline
\end{tabular}

\section{DISCUSSION}

The purpose of this study was to investigate the effects of audio biofeedback on the jump height of figure skaters. Previous literature on figure skating has failed to demonstrate increases in jump height, a factor considered crucial for the success in the sport. It was 
hypothesized that audio biofeedback would increase the jump height and knee flexion during the off-ice Lutz jump. The hypothesis was partially supported.

The results of the present study indicate that auditory biofeedback has the potential to modify motor performance of the off-ice Lutz jump. These findings are in line with previous studies that have demonstrated that auditory biofeedback can affect motor performance of complex movements like posture and balance ${ }^{7,8,9,10,11,12,13}$ and cycling performance in children. ${ }^{14}$ In the present study, the use of auditory biofeedback during the performance of off-ice Lutz jumps decreased time in the air, increased knee flexion, and had no effect on EMG activity of the rectus femoris. However, it had been hypothesized that audio biofeedback would increase the jump height and knee flexion during the off ice Lutz jump. That hypothesis was partially confirmed.

Previous research has shown that increased knee flexion was fundamental for increasing jump height. ${ }^{15,16}$ During trials with auditory biofeedback, participants indeed had increased knee flexion. The increase in knee flexion did not, however, lead to increased jump height. In fact, the opposite occurred. During the trials with auditory biofeedback the skaters had greater knee flexion but shorter times in the air. One potential reason for the negative effect of audio biofeedback on time in the air is it might have led participants to adopt an internal focus of attention.

As individuals become more skilled in a particular task, the amount of attention deliberately directed to performing that task is reduced. ${ }^{17}$ As such, experts require little to no attention devoted specifically to skill execution to successfully perform a movement. In support of this, there exists a growing body of evidence that indicates that directing the performer's attention to his or her own body (i.e. internal focus of attention) instead of the environment (i. e. external focus) disrupts performance by forcing control instead of allowing the nervous system to reinforce its automatic control of movements. ${ }^{18}$ This body of research argues that an internal focus of attention constrains the performer and increases noise in the motor system, thus degrading performance. ${ }^{19,20}$ Wulf et al. (2010), for example, found that participants performing a vertical jump-and-reach task under and internal and external focus condition in a counterbalanced order demonstrated higher EMG activity and lower jump height under the internal focus condition. Though the present study did not specifically investigate the effects of different attentional foci, these results appear in line with the findings of the present study.

Furthermore, the disruption in the figure skaters' performance may have occurred in the spatio-temporal characteristics of the jump. It is speculated that during trials with auditory biofeedback, skaters may have performed the flexion of the knee, reached the lowest point of the takeoff portion of the Lutz jump, and then paused very briefly to "wait" for the auditory biofeedback signal to receive confirmation that the performer had reached the appropriate knee flexion and that it was then time to perform the jump.

The different times in the air for the conditions in the present study could be seen as analogous to the differences observed between countermovement vertical jumps and squat vertical jumps. During countermovement jumps, performers start from a standing position and move downwards before pushing off into a jump. During squat jumps, performers start from a semi squat position and perform no countermovement prior to pushing off into a jump. Generally, performers achieve greater jump height in countermovement jumps compared to squat jumps. ${ }^{22}$ The pause that may have occurred during the trials with auditory biofeedback in the present study may have created conditions similar to those observed in the squat jump, thus explaining the lower time in 
the air during the trials with auditory biofeedback. Although the time delay between the beep from the audio biofeedback device and initiating the jump was not directly measured, it prompts future research to indicate whether it is indeed a factor in influencing jump height and time in the air.

In conclusion, the present study demonstrated that auditory biofeedback has the potential to modify motor performance in the off-ice single Lutz jump, as indicated by the increase in knee flexion. The changes observed however were not expected. During trials with auditory biofeedback, skaters had lower times in the air, possibly because auditory biofeedback lead participants to adopt an internal focus of attention, which lead to decrements in performance through mechanisms previously described by Wulf (2007). Though the study contributes to the literature on the effectiveness of auditory biofeedback and to the limited literature ${ }^{4,5}$ on enhancing performance of figure skaters, there are a few limitations that should be addressed by future studies. In the present study the target muscle activity of the rectus femoris was determined during a static position. That clearly does not reflect the dynamic demands of the single Lutz jump. Future studies should explore different ways to determine target muscle activation. Another possible study should investigate the use of auditory biofeedback in less skilled figure skaters. It is possible that for novices, who require more attention to perform a task ${ }^{17}$, would not experience the disruption observed in the study since they potentially are already paying attention internally. Less skilled skaters would also avoid any potential ceiling effects observed in the present study. Also, it is recommended to include a verification mechanism for the focus of attention adopted by the participants. ${ }^{23}$ This would allow a greater understanding of the mechanisms governing the effects of auditory biofeedback manipulations.

\section{REFERENCES}

1. King, D.L. (2005). Performing triple and quadruple figure skating jumps: Implications for training. Can J Appl Physiol, 30(6), 743-753.

2. King, D., Smith, S., Higginson, B., et al. (2004). Characteristics of triple and quadruple toeloops performed during The Salt Lake City 2002 Winter Olympics. Sport Biomech, 3(1), 109-123.

3. Albert, W.J., \& Miller, D.I. (1996). Takeoff characteristics of single and double axel figure skating jumps. J Appl Biomech, 12, 72-87.

4. Law, B., \& Ste-Marie, D.M. (2005). Effects of self-modeling on figure skating jump performance and psychological variables. Eur J Sport Sci, 5(3), 143-152.

5. Haguenauer, M., Fargier, P., Legreneur, P., et al. (2005). Short-term effects of using verbal instructions and demonstration at the beginning of learning a complex skill in figure skating. Percept Mot Skills, 100, 179-191.

6. Moss, D., Sella, G.E., Andrasik, F., et al. (2003). Current applications of biofeedback to physical medicine and rehabilitation. Eur Med, 39, 165-170.

7. Chiari, L., Dozza, M., Cappello, A., et al. (2005). Audio-biofeedback for balance improvement: An accelerometry-based system. IEEE Trans Biomed Eng, 52(12), 21082111. 
Brazilian Journal of Motor Behavior

8. Dozza, M., Chiari, L., Chan, B., et al. (2005). Influence of a portable audio-biofeedback device on structural properties of postural sway. J Neuroeng Rehabil, 2(13).

9. Dozza, M., Chiari, L., Hlavacka, F., et al. (2006). Effects of linear versus sigmoid coding of visual or audio biofeedback for the control of upright stance. IEEE Trans Neural Syst Rehabil Eng, 14(4), 505-512.

10. Dozza, M., Horak, F.B., \& Chiari, L. (2007). Auditory biofeedback substitutes for loss of sensory information in maintaining stance. Exp Brain Res, 178(1), 37-48.

11. Dozza, M., Chiari, L., Peterka, R.J., et al. (2011). What is the most effective type of audiobiofeedback for postural motor learning? Gait \& Posture, 34(3), 313-319.

12. Mierlman, A., Herman, T., Nicolai, S., et al. (2011). Audio-biofeedback training for posture and balance in patients with Parkinson's disease. J Neuroeng Rehabil, 8(35).

13. Nicolai, S., Mierlman, A., Herman T., et al. (2010). Improvement of balance after audiobiofeedback. Z Gerontol Geriatr, 43, 224-228.

14. Liu, T., \& Jensen, J.L. (2009). Effectiveness of auditory and visual sensory feedback for children when learning a continuous motor task. Percept Mot Skills, 109(3), 804-816.

15. Moran, K.A., \& Wallace, E.S. (2007). Eccentric loading and range of knee joint motion effects on performance enhancement in vertical jumping. Hum Mov Sci, 26, 824-840.

16. Vanezis, A., \& Lees, A. (2005). A biomechanical analysis of good and poor performers of the vertical jump. Ergonomics, 48, 1594-1603.

17. Fitts, P.M., \& Posner, M.I. (1967). Human Performance. Oxford; England: Brooks/Cole.

18. Wulf, G., Tollner, T., \& Shea, C.H. (2007). Attentional focus effects as a function of task difficulty. Res Q Exerc Sport, 78(3), 257-264.

19. Vance, J., Wulf, G., Tollner, T., et al. (2004). EMG activity as a function of the performer's focus of attention. J Mot Behav, 36(4), 450-459.

20. Zachary, T., Wulf, G., Mercer, J., et al. (2005). Increased movement accuracy and reduced EMG activity as the result of adopting an external focus of attention. Brain Res Bull, 67, 304-309.

21. Wulf, G., Dufek, J.S., Lozano, L., et al. (2010). Increased jump height and reduced EMG activity with an external focus. Hum Mov Sci, 29(3), 440-448.

22. Bobbert, M.F., Gerritsen, K.G., Litjens, M.C., et al. (1996). Why is countermovement jump height greater than squat jump height? Med Sci Sports Exerc, 28, 1402-1412.

23. Post, P.G., Barros, J.A.C., \& Wrisberg, C.A. (2011). Evidence of non-compliance with instructions in attentional focus research. Brazilian Journal of Motor Behavior, 6(1), 1-6.

Citation: Barros JAC, Florendo L, Le Y (2014) Auditory Biofeedback Decreases Jump Performance In Figure Skaters. BJMB 8 (1).

Editor: Marcio A. Oliveira, University of Maryland, College Park, MD, USA.

Copyright: (c) 2014 Barros et al and BJMB. This is an open-access article distributed under the terms of the Creative Commons Attribution-NonCommercial-NoDerivatives 4.0 International License which permits unrestricted use, distribution, and reproduction in any medium, provided the original author and source are credited.

Funding: This study was not supported by any fellowship or funding.

Competing interests: The authors have declared that no competing interests exist.

Download: http://socibracom.com/bjmb/index.php/bjmb/issue/view/10 